\title{
How can human motion prediction increase transparency?
}

\author{
Nathanaël Jarrassé, Jamie Paik, Viviane Pasqui and Guillaume Morel
}

\begin{abstract}
A major issue in the field of human-robot interaction for assistance to manipulation is transparency. This basic feature qualifies the capacity for a robot to follow human movements without any human-perceptible resistive forces.

In this paper we address the issue of human motion prediction in order to increase the transparency of a robotic manipulator. Our aim is not to predict the motion itself, but to study how this prediction can be used to improve the robot transparency. For this purpose, we have designed a setup for performing basic planar manipulation tasks involving movements that are demanded to the subject and thus easily predictible. Moreover, we have developed a general controller which takes a predicted trajectory (recorded from offline free motion experiments) as an input and feeds the robot motors with a weighted sum of three controllers: torque feedforward, variable stiffness control and force feedback control.

Subjects were then asked to perform the same task but with or without the robot assistance (which was not visible to the subject), and with several sets of gains for the controller tuning. First results seems to indicate that when a predictive controller with open loop torque feedforward is used, in conjunction with forcefeeback control, the interaction forces are minimized. Therefore, the transparency is increased.
\end{abstract}

Index Terms-Interactive robotics, transparency, position/force control.

\section{INTRODUCTION}

In various new applications of interactive robotics, which range from haptics to force-feedback telemanipuation, from fine surgical gesture assistance to rehabilitation, a robotic device and a human simultaneously manipulate the same object. In most of these applications, the robot is programmed to exert forces and/or to follow a trajectory with the aim of helping the subject to perform a manipulation task.

One of the performance indexes that quantify the robot ability to precisely produce a programmed assistance to the subject is its transparency. This may seem contradictory, since transparency measures the robot ability of not applying any assistance. In fact, transparency is a good indicator for force precision since any failure to reach transparency during a zero-resistance experiment will be reproduced and act as a bias in a non-zero force experiment.

The first notable research effort of transparency is seen in the field of haptic devices in its mechanical design. In this domain, a particular care shall be put in reducing joint friction and end-effector inertia, which is usually antagonistic with the ability of producing large forces. Achieving transparency for an assisting device of upper limb movements requires several specifications: an important workspace without singularities, a complete reversibility and a low inertia as well as force feedback capacities and stiffness [1]. Moreover, the stability

The authors are with the Univsersit P. et M. Curie, Insitut des Systèmes Intelligents et de Robotique (CNRS - FRE 2507). Email: \{jarrasse, paik, pasqui, morel\}@robot.jussieu.fr. needed for this kind of robots, manipulated by humans, limits the controller stiffness [2]. Recently, new design technologies were developed, which greatly enhance transparency, like those developed by the CEA (French center for atomic energy) for nuclear remote controlled manipulators. A new kind of actuator using ball screw and cable transmission for a large reduction ratio and a good reversibility is proposed in [3]. Another recent example of highly transparent device is the McGill University Pantograph [4]. Here, transparency is achieved in a limited workspace with a reduced number of degrees of freedom, due to its planar parallel mechanical structure.

In any cases, friction and inertia, which are unavoidable, limit the overall system bandwidth and its transparency. Therefore, real time active control shall be considered as a mean of overcoming these limits.

Including a force sensor and implementing force feedback control is the most popular solution to this problem. The force sensor shall be mounted at the precise place where transparency is needed, usually between the wrist and the end effector for a serial manipulator. Force feedback control allows to cancel quite easily the static joint friction phenomenon. However, it suffers from several limitations : stability, drift, bandwidth limitations. In addition to discrete control problems and sensor noise, dynamics between actuators and force sensors drastically limits force controller performances [2]. Bandwidth limitations are the major problem of these controllers [5], which in turn address the antagonisms of the design such as rigidity vs inertia and friction.

Several recent papers discuss advanced way of tackling the human-robot interaction problems. Buerger and Hogan [6] suggest a new approach to improve performance and stability of robot controllers based on force feedback. By studying the differences with classical servo control problems, they introduce new control design tools dedicated to the human interaction problem. They have shown a better performing controller by using the environmental information on dynamics to transform a coupled stability problem into a robust stability one.

Some other research work concerns overcoming force control bandwidth limitations with a new approach based on predictions of the subject's intended movement. The well known control principle is to overcome the force closed loop precision in spite of bandwidth limitations with the feedforward loop.

A major topic in this domain is to predict human movement. Several invariant characteristics in human movement exist along with a few movement laws, especially dedicated to the upper limb: bell-shaped speed profile [7] (which is linked to the well known minimum jerk criterion [8]), linear 
synergy between the joints [9], isochrony (relation between trajectory length and movement speed), Fitts law (describing the speed/accuracy compromise) or the power law (relation between speed and trajectory curvature) [10]. All these laws may be used to reconstitute in real time the characteristics of a movement. Typically, one can predict the end of the movement from very little information retrieved from sensors at the beginning of a movement. For a simple point-to-point movement, the knowledge of the trajectory beginning is almost enough to reconstitute the whole trajectory with the minimum jerk criterion [11]. Other technical means could be also used to predict human arm movement: Saccadic eye movements or gaze tracking can help detecting movement initiation [12] and predicting the future movement [13]. Human-robot cooperation in an assembly task based on human intention interpretation from gaze movement has been recently tested [14]. By adapting neural networks, electromyograms (EMG) signals were successfully used to predict arm movement along with arm forces[15] or to control in a predictive way an exoskeleton leg orthosis [16].

Assuming that a motion prediction can be done with enough precision, cooperative robot control can definitely benefit from this information. Corteville et al. [11] developed a robot assistant for fast point-to-point movement inspired by human motion models. Their one DOF robot reacts to human forces imposed on a handle (with an admittance controller),while identifying the motion to move along with the operator, in order to make the movement more comfortable and natural (transparent) to the subject. This method proves that active participation of the robot based on a model of human movement is advantageous. However, the use of an admittance controller as the lowest level of the controller architecture does not seem to be pertinent. Indeed, the benefits of the force loop and human movement prediction are badly impacted by the drastically low bandwidth of the inner position loop. Therefore, the overall transparency is limited, involving large forces at the human-machine interaction port. Duchaine and Gosselin [17] have recently developed a similar controller with the capacity of understanding human intention with a force sensor application. A low level velocity controller is exploited instead of a position controller. This approach is based on an online variable impedance control. During the comanipulation task, the controller impedance is permanently adapted to the subject movement, according to the time derivative of the force. The experimental validation was made by executing a drawing task with a parallel manipulator which showed evidences of increased transparency.

The general idea developed in this paper is to exploit a high bandwidth low level controller in combination with a feedforward compensator based on a human motion prediction. It takes the form of a controller combining a joint position compensator, a feedforward trajectory tracking, and a direct force feedback term, which is covered in Section II. An experimental platform was then set up to evaluate this controller. Recall that our aim is not to predict movement, but to understand how to use this prediction at the control level.
This is why a specific experimental protocol was defined (see Section III: first, we record several movements of a subject repeatedly to realize a free planar reaching task; an averaged data set extracted from the free reaching tasks is then used as a prediction during the transparency experiment. The transparency experiments consist for a given human subject in repeating the same movement while being attached to a robot, while several combinations of the three control strategies are combined. Meanwhile, transparency is evaluated (i.e. the force magnitude is measured) and subject feelings are monitored. The experimental results obtained with a limited number of subjects are presented and compared in Section IV. Finally in Section IV, we discuss about the impact of introducing human motion prediction into transparency control and about the further experiment for endorsing and generalizing these first results.

\section{HUMAN MOTION PREDICTION-BASED TRANSPARENCY CONTROL}

In this section we derive a general control structure aimed at increasing the transparency of a robotic device held by a human subject based on a prediction of the subject's movement. It is assumed that a prediction is available, which takes the form of a robot joint trajectory, $\mathbf{q}_{d}(t)$. The initial time of the movement, $t_{0}$, is also supposed to be known. The really intended motion of the operator, parameterized in the joint space is denoted $\mathbf{q}_{r}(t)$. It is the motion that the subject would produce without any robot connected to his/her hand. A perfect prediction is thus characterized by $\mathbf{q}_{d}(t) \equiv \mathbf{q}_{r}(t)$.

Furthermore, the robotic device is supposed to be governed by the following dynamical equation :

$$
\Gamma_{m}+\mathbf{J}^{\mathrm{T}}(\mathbf{q}) \mathbf{F}_{e x t}=\mathbf{H}(\mathbf{q}) \ddot{\mathbf{q}}+\mathbf{b}(\mathbf{q}, \dot{\mathbf{q}})+\mathbf{g}(\mathbf{q})+\Gamma_{f},
$$

where $\Gamma_{m}$ is the motor torque resulting from the current, $\mathbf{J}(\mathbf{q})$ is the robot jacobian matrix describing the kinematic mapping from the joint space to the end-effector space, $\mathbf{F}_{e x t}$ is the external wrench applied by the operator, $\mathbf{H}(\mathbf{q})$ is the joint space inertia matrix, $\mathbf{b}(\mathbf{q}, \dot{\mathbf{q}})$ regroups the Coriolis and centrifugal effects, $\mathbf{g}(\mathbf{q})$ is the joint torque of gravity, and $\Gamma_{f}$ is the joint friction torque.

Several control strategies can be applied. The first control strategy that we have implemented is force feedback control, by the use of a joint-level torque compensator $\mathbf{C}_{f}$ :

$$
\Gamma_{m, 1}=-\mathbf{C}_{f}\left[\mathbf{J}^{\mathrm{T}}(\mathbf{q}) \mathbf{F}_{e x t}\right],
$$

where $\mathbf{J}^{\mathrm{T}}(\mathbf{q})$ is used to map the measured force $\mathbf{F}_{\text {ext }}$ into a joint equivalent. Note that this controller does not benefit from any predicted motion, it is only reactive to subject forces. In fact, it will be used as the reference for the force minimization performance during the experiments.

The second option is a trajectory control, for which the robotic device is programmed to precisely follow the desired trajectory $\mathbf{q}_{d}(t)$, with a joint position compensator $\mathbf{C}_{p}$ :

$$
\Gamma_{m, 2}=\mathbf{C}_{p}\left[\mathbf{q}_{d}(t)-\mathbf{q}(t)\right] .
$$


Note that the compensator may include an estimated dynamical model of the robot, e.g. it may realize a dynamical decoupling due to the set of estimated parameters $\hat{\mathbf{H}}(\mathbf{q}), \hat{\mathbf{g}}(\mathbf{q})$, $\hat{\mathbf{b}}(\mathbf{q}, \dot{\mathbf{q}}), \hat{\Gamma}_{f}$. Ultimately, if both the prediction and the robot dynamic positioning precision were perfect, then the robot and the subject would produce the exact same motion (i.e. $\left.\mathbf{q}(t) \equiv \mathbf{q}_{d}(t) \equiv \mathbf{q}_{r}(t)\right)$. This would result in no dynamic forces at the interface. However, we do not expect this strategy to be robust with respect to the motion prediction errors. Indeed, in order to achieve a high precision, it is required that high gains are used. Therefore, if $\mathbf{q}_{d}(t)$ differs (even slightly) from the real subject intended motion $\mathbf{q}_{r}(t)$, which seems unavoidable, then large forces will occur at the interface. This controller is still kept as a candidate in order to experimentally evidence this expected phenomenon.

The third strategy that can be implemented is a feedforward trajectory tracking:

$$
\Gamma_{m, 3}=\hat{\Gamma}_{m}\left(\mathbf{q}_{d}, \dot{\mathbf{q}}_{d}, \ddot{\mathbf{q}}_{d}\right),
$$

where $\hat{\Gamma}_{m}\left(\mathbf{q}_{d}, \dot{\mathbf{q}}_{d}, \ddot{\mathbf{q}}_{d}\right)$ is the estimation of the torque that the actuator shall produce in order to follow the desired trajectory. Note that possible realization of the torque feedforward is:

$$
\hat{\Gamma}_{m}\left(\mathbf{q}_{d}, \dot{\mathbf{q}}_{d}, \ddot{\mathbf{q}}_{d}\right)=\hat{\mathbf{H}}\left(\mathbf{q}_{d}\right) \ddot{\mathbf{q}}_{d}+\hat{\mathbf{b}}\left(\mathbf{q}_{d}, \dot{\mathbf{q}}_{d}\right)+\hat{\mathbf{g}}\left(\mathbf{q}_{d}\right)+\hat{\Gamma}_{f}
$$

Again, with a perfect prediction and a perfect torque estimation, one gets $\mathbf{q}(t) \equiv \mathbf{q}_{d}(t) \equiv \mathbf{q}_{r}(t)$. Moreover, with this approach, in contrary to the first strategy, small discrepancy between the predicted and real motions will not produce high forces at the interface. This is why we expect this approach to provide a better feeling of the transparency.

In the rest of the paper, the controller will be a weighted sum of the three strategies described in Equations (2), (3) and (4):

$$
\Gamma_{m}=\alpha_{1} \Gamma_{m, 1}+\alpha_{2} \Gamma_{m, 2}+\alpha_{3} \Gamma_{m, 3},
$$

where $\alpha_{i} \in[0,1]$, for $i \in\{1 . .3\}$. Tuning the parameters $\alpha_{i}$ is a way of applying the different strategies, alone or in combination.

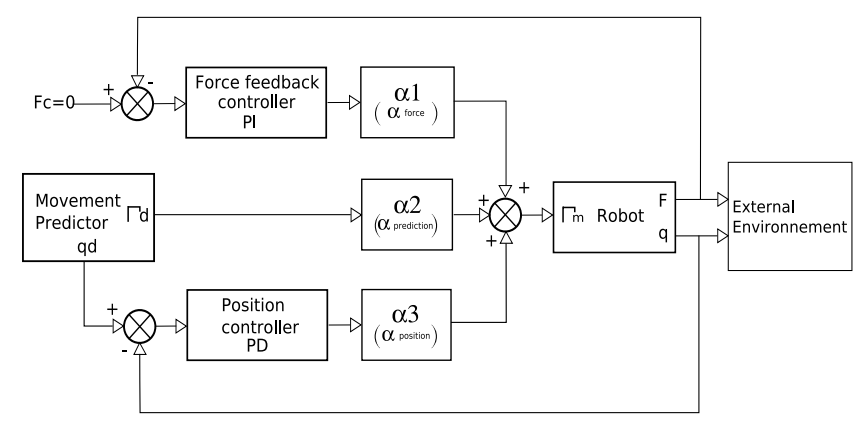

Fig. 1. Three strategies controller

\section{EXPERIMENTAL SETUP}

The elementary planar manipulation tasks were performed with a specific handle fitted with position and force sensors, which can be used alone or mechanically connected to the end-effector of a haptic Interface (Virtuose 3D, Haption). This device possesses 3 active joints and 3 passive joints forming a very low friction wrist sensors. sensors).

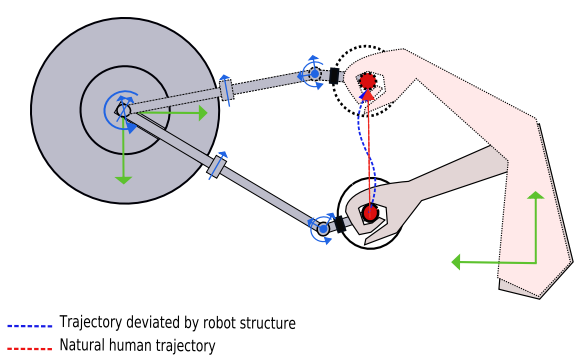

Fig. 2. Simple point-to-point movement

An opaque surface was installed hiding the hand of the subject, so that the availability of the robot cannot be known and hinder the experimental results: with this apparatus, the subject attention is focused on this white planar surface, while he/she waits for the start signal given through an LED to reach a target materialized on the surface by a circle. In order to allow the subject to see his/her hand position through the opaque surface placed over the table and the robot, a laser pointer is placed inside the handle and projects a spot on the surface. Starting and ending ocations are always visible over the opaque surface throughout the experiment.

The experiment begins by telling the subject to grab the handle (disconnected from the robot) which is placed under the opaque surface. Then he is instructed to perform five times the same simple point-to-point movement, meanwhile the handle position is recorded. The measures are then averaged and filtered in order to synthesize a movement model of the subject trajectory, later used as a prediction for the transparency experiment.

The transparency experiment consists in performing the same point to point movement with varying controller configurations. The transparency evaluation is made by norm of the interaction force during the task.

After the trajectory recording part, the subject is told to perform the same movement when the red LED turns on.No particular speed indication and/or way of grasping the handle during the movement were instructed so that the test results are that of the most natural behaviour of human subject.

\section{A. Robotic device}

The experimental campaign was performed with a Virtuose manipulator from Haption, which has a three active degrees of freedom and a passive wrist possessing three rotation axes intercepting at point $W$. This kind of kinematic configuration allows us to ease the control while enabling, unlike a singledimensional system, unconstrained natural human movement during a co-manipulation task. This haptic interface system is actuated by three 90 Watts Maxon DC motors fitted with wire reducers. This setup generates a $15 \mathrm{~N}$ force peak at point $W$ (where the moment is 0 ) and a $5 \mathrm{~N}$ continuous force. The 
stiffness of the device is about $800 \mathrm{~N} / \mathrm{m}$. The angular motor position is measured by optical incremental encoders for the three active DOFs and by potentiometers for the DOFs of the wrist. The handle is mounted on the end effector extremity. Between the handle and the effector, a force sensor is installed in order to measure the force exerted by the subject on the robot and inversely. This measured force is used to compute the control law (2) where the Jacobian is computed at point $W$.

The 6-axis force/torque(F/T) sensor is an ATI Nano43 Transducer allowing us to reconstruct the 3 forces and 3 torques components. For the experiments we are only interested in two of the six components (X and $\mathrm{Y}$ force components) and for this component the force range is $+/-36 \mathrm{~N}$ with a resolution of about $1 \mathrm{mN}$. The controller is equipped with an Analog and Digital I/O PCI card (National Instrument, model 6034E) in which we use six 16 bit A/D channels for acquiring the readings of the force sensor.
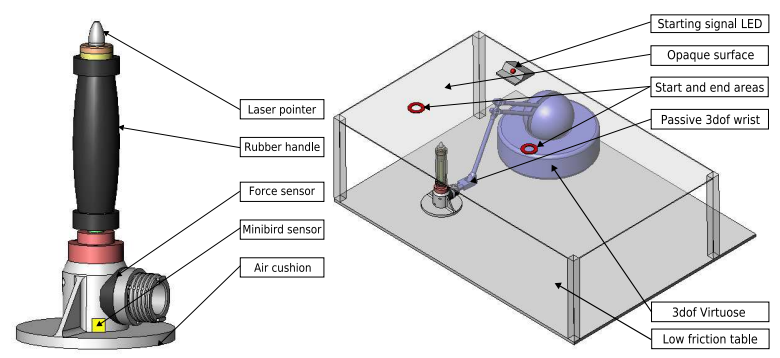

Fig. 3. 3D view of the handle and of the experimental setup

The handle is also fitted with a magnetic position and orientation sensor (Minibird, Ascension Technology), which is installed under the force sensor, the fixed magnetic emitter being placed under the table. It provides position and orientation measurements at a $100 \mathrm{~Hz}$ frequency and compute speed of the handle during the experiments. It allows the controller to learn movement characteristics of the subject during the preexperimental part, but is not used during the transparency tests. As the experiment deals with low level-forces, a particular attention has been given to minimize friction. This is why the lowest part of the handle was designed with an air cushion system, in the purpose of reducing friction between the handle and the table, in case the subject strongly pushes against the sliding surface.

The robot controller architecture is based on a PC104 board with an endowed 3 channel axis controller. It runs at $1 \mathrm{kHz}$ the control law 6 thanks to a real time operating system (RTlinux).

\section{B. Trajectory recording}

During the pre-experiments, the subject is asked to perform the same movement from the start area to the end area (marked up over the opaque surface by $3 \mathrm{~cm}$ diameter circles). This is repeated five times in a row. Five attempts are enough to extract general features of the subject movement, as it was experimentally verified that healthy subjects performing free upper-limb movements produce quite repeatable motions.
Data is filtered and then interpolated from $100 \mathrm{~Hz}$ record (maximum data rate of the minibird sensor) to a $1 \mathrm{kHz}$ data trajectory compatible with the control loop clock.

Another important data is the reaction time of the subject (the time laps needed by a subject to initiate the movement after the visual start signal is turn on). Indeed, the "anticipation" is done by reinjecting a recorded characteristic move.It is thus important to perfectly synchronize when the subject starts to move and the point of the recorded motion $\mathbf{q}_{d}$ starts. The knowledge of that reaction time $t_{0}$ is made during the learning phase and allows us to synchronize robot anticipation with the subject move during the evaluations experiments. Figure 4 shows the result for a representative subject. It can be noticed that, since the hand is not seen during the experiments, the initial acceleration is much larger than the final deceleration (when the subject carefully manipulates to reach the target zone). Therefore, interaction forces are expected to be larger at the movement initialization than at the final motion during robot aided experiments.
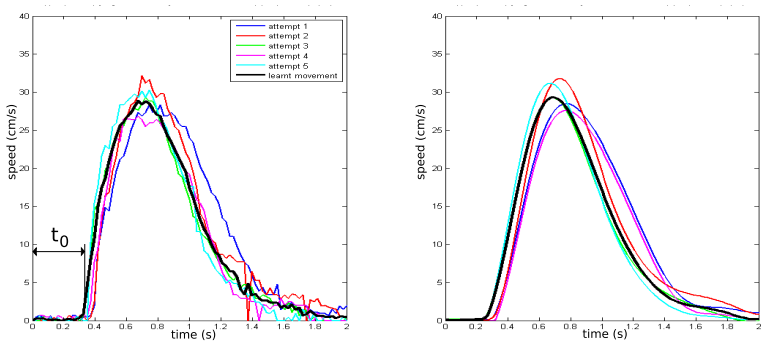

Fig. 4. Graphics of measured and interpolated speeds for the same simple point-to-point movement for one subject

\section{Computing $\hat{\Gamma}_{m}$}

Calculating $\hat{\Gamma}_{m}$ with equation (5) would require a model parameter identification and lead to imprecision. We have used a simple experimental method which had the double advantage of good precision and no model requirement.

Once the trajectory $\mathbf{q}_{d}(t)$ is available, the robot end-effector extremity is placed on the start area with a standard PD position controller (see Eq. 3). Then the recorded interpolated average trajectory is fed to the robot controller. During the robot movement, the motor currents are recorded. In fact, during this experiment, the position control loop calculates the necessary torques to apply to actuators to move the robot structure along the human subject trajectory. The resulting output is $\hat{\Gamma}_{m}$, which will be used as an open-loop feedforward signal to realize the prediction feature of the controller.

\section{RESUlts OF TRANSPARENCY TESTS}

During the experiments we used a PD compensator for the position controller which was tuned manually to provide satisfactory trajectory tracking, and a PI compensator for the force control loop, which was tuned manually to perform stably and fast enough in the whole workspace. For each subject, we evaluated interaction forces on the handle for a 
simple point-to-point movement with $\alpha_{1}=0$ or $1, \alpha_{2}=0$ or 0.2 or 1 , and $\alpha_{3}=0$ or 1 , as depicted in Table I. The experiments were performed in a random order.

\begin{tabular}{||l|l|l|l|l|c|c|c|c|c||}
\hline Experiment \# & 1 & 2 & 3 & 4 & 5 & 6 & 7 & 8 & 9 \\
\hline$\alpha_{1}$ & 0 & 1 & 0 & 1 & 0 & 1 & 0 & 1 & 1 \\
\hline$\alpha_{2}$ & 0 & 0 & 1 & 1 & 0.2 & 0.2 & 0 & 0 & 0.2 \\
\hline$\alpha_{3}$ & 0 & 0 & 0 & 0 & 0 & 0 & 1 & 1 & 1 \\
\hline
\end{tabular}

TABLE I

GAINS USED FOR THE 9 EXPERIMENTS

Our references during the experiments, in terms of the magnitude of forces at the interaction port, are shown in Experiment 1 , where the null current is applied on the robot during the move, so that only the residual friction of the haptic device together with its inertia are felt. In Experiment 2, where force feedback controller is used alone $\left(\alpha_{1}=1\right.$ and $\alpha_{2}=\alpha_{3}=0$ ). We show in Figure 5 the norm of the planar force, $f=\sqrt{f_{x}^{2}+f_{y}^{2}}$, which is observed during these two experiments for a representative subject.

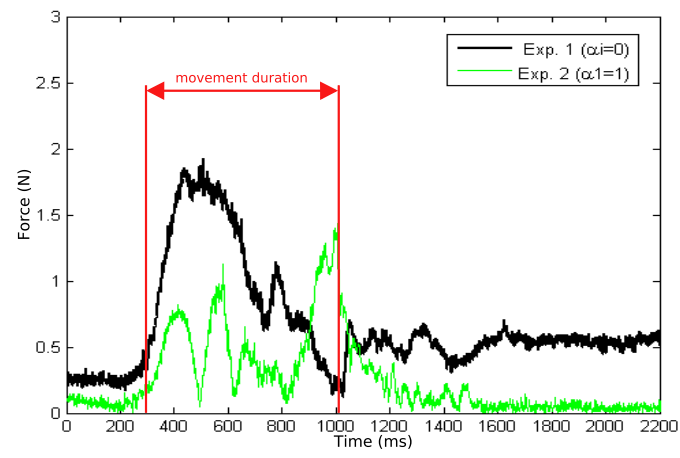

Fig. 5. Norm of the force at the interface during point-to-point movement with null current (EXP1) and force feedback controller alone (EXP2)

As expected, force feedback control provides a good level of transparency by limiting the forces during the experiment. Regardless of the level of exchanged forces, we can observe a force peak at the start of the move. Indeed, the beginning of the motion requires sudden large forces to initiate movement and the force feedback controller is finally slow to react due to its bandwitdh limitations. In spite of the good level of performance obtained by the reversible haptic device and a low level force feedback controller with a decent bandwidth, these experiments lead us to think that we can continue minimizing the interaction force by using the trajectory prediction in the controller.

The first way to exploit this predictive information is to use a rigid joint position compensator $\left(\alpha_{2}=1\right)$. We thus performed two experiments: Experiment 3 involves the trajectory controller alone $\left(\alpha_{2}=1\right.$ and $\left.\alpha_{1}=\alpha_{3}=0\right)$ while Experiment 4 simultaneously uses position and force control by setting $\alpha_{1}=\alpha_{2}=1$ and $\alpha_{3}=0$. As we can observe in Figure 6, the use of a rigid joint position compensator alone leads, as expected, to large forces at the interface. Of course, the force controller added in Experiment 4 compensates for this effect, but the

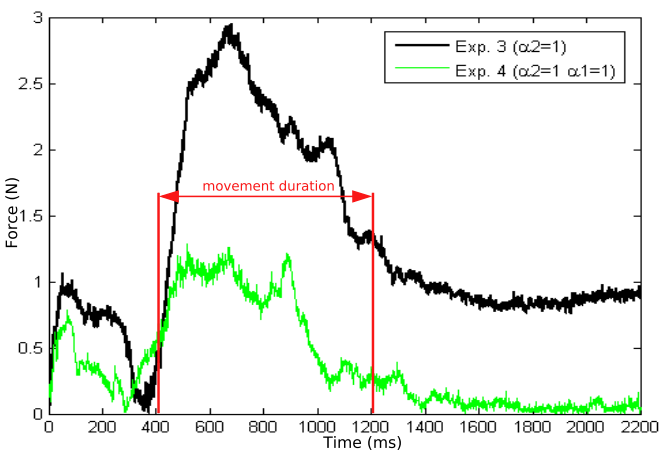

Fig. 6. Norm of the force at the interface for Experiments 3 and 4

result of this experiment, when compared to Experiment 2 where force sensor is used alone, shows that using $\alpha_{2}=1$ is of no interest.

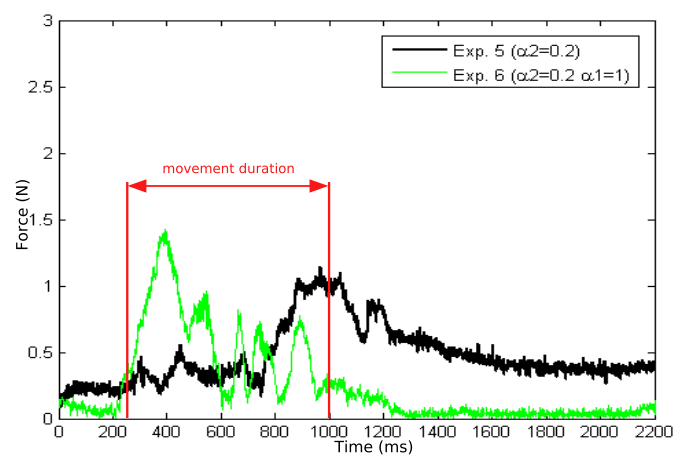

Fig. 7. Norm of the force at the interface for Experiments 5 and 6

We supposed that this is mostly due to the rigidity of the controller, and therefore proposed to run two new experiments similar to Experiments 3 and 4 while $\alpha_{2}$ was set to 0.2 . The results for Experiments 5 and 6 are plotted in Figure 7. When compared to the results of Experiments 1 and 2, respectively, they emphasize a clear decrease of the average force. Experiment 5, in particular, is a good clue that the transparency can be increased through low stiffness position tracking when no force sensor is available.

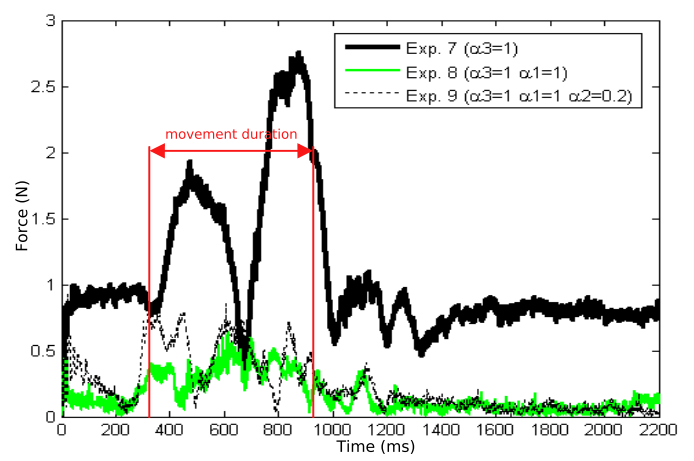

Fig. 8. Norm of the force at the interface for Experiments 7 to 9

The last set of experiments concerned the use of a feedforward 
torque resulting from the desired trjectory. Three experiments were performed: feedforward alone (Exp. 7), feedforward plus force feedback control (Exp. 8) and feedforward plus force feedback control plus low stiffness trajectory tracking (Exp. 9). Results for these three experiments are given in Figure 8. We can see that, when the feedforward term is used alone, the operator badly reacts to the robot open loop activity, which leads to rather large interaction forces. However, when force control is used in conjunction with the feedforward term, a clear performance increase is observed as compared to reference Exp. 2. This confirms the interest of predicting the motion for increased transparency, with a technical solution that is alternative to a low stiffness position tracking. Moreover, Exp. 9 , using the combination of force feedback, feedforward, and low stiffness position tracking, exhibits results that are as good as Experiment 8. In Figure 9,the graphs summarize the results of the nine experiments, clearly shows that the average forces are minimized for Exp. 8 and 9.

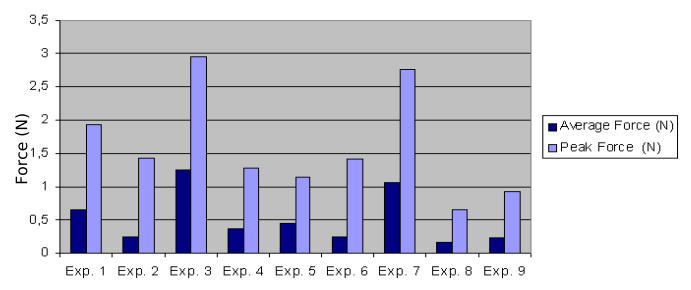

Fig. 9. Peak force and average force during the nine experiments

Figures 5 to 9 display the results for a single subject that cannot be generalized. However, the general tendencies among the six subjects are depicted hereafter.

This early work seems to show that human motion prediction, even if it's not sufficient alone, could allow force controllers to achieve a better transparency than non predictive strategies. This is consistent with several published studies mentionned in the introduction, such as [11].

Quite interesting clues can be extracted from the current results. The use of feedforward trajectory tracking seems rewarding in terms of transparency, with a real efficiency at the beginning of the move. This is consistent with Corteville's note [11] regarding the segmentation of a point to point movement into several phases. Our results indicate that the controller could split the strategy along a trajectory in two parts:

1) The beginning and the ending of the motion requires large forces to initiate and stop movement (to accelerate the entire robot structure, to overcome dry friction, and to decelerate) which is difficult to compensate by using force feedback alone. That's why the addition of feedforward trajectory tracking (or a limber joint position compensator) produces the best transparency.

2) In the middle of the trajectory, very little forces are needed, human haptic sensibility is thus enhanced. Even a little desynchronization between the applied anticipation and the real movement may be disturbing to the subject. Moreover, the acceleration is small which limits the force error due to the bandwidth limitation. That is why during this second phase, the force feedback seems to be enough to maximize transparency at the interface. Therefore it could be interesting to use time varying $\alpha_{i}(t)$ in order to maximize the predictive strategy at the beginning and the end of the motion, and minimize the effects during the rest of the movements.

We leave this to future investigations, which shall also include a statistical results analysis based on a larger number of subjects.

\section{REFERENCES}

[1] Thomas H. Massie and J. K. Salisbury. The phantom haptic interface: A device for probing virtual objects. In Proceedings of the ASME Winter Annual Meeting, Symposium on Haptic Interfaces for Virtual Environment and Teleoperator Systems, Nov 1994.

[2] J.E. Colgate and J.M Brown. Factors affecting the z-width of a haptic display. In Robotics and Automation, 1994. Proceedings., 1994 IEEE International Conference on, volume 4, pages 3205-3210, May 1994.

[3] P. Garrec, JP. Martins, and JP. Friconneau. Une nouvelle technologie d'orthese portable. Handicap 2004, June 2004.

[4] G. Campion, Q. Wang, and V. Hayward. The pantograph mk-2: A haptic instrument. IROS 2005, IEEE/RSJ Int. Conf. Intelligent Robots and Systems, pages 723-728, 2005.

[5] S. Eppinger and W Seering. Understanding bandwidth limitations in robot force control. In Robotics and Automation. Proceedings. 1987 IEEE International Conference on, volume 4, pages 904-909, Mar 1987.

[6] S.P. Buerger and N. Hogan. Complementary stability and loop shaping for improved human-robot interaction. In Robotics, IEEE Transactions on [see also Robotics and Automation, IEEE Transactions on], volume 23, pages 232-244, 2007.

[7] Gordon J., Ghilardi MF., and Ghez C. Accuracy of planar reaching movements. independence of direction and extent variability. Exp Brain Res, 1:97-111, 1994.

[8] Flash T. and Hogan N. The coordination of arm movements: an experimentally confirmed mathematical model. The Journal of Neuroscience, 5, Jul 1985

[9] G. L. Gottlieb, Q. Song, D. A. Hong, and D. M. Corcos. Coordinating two degrees of freedom during human arm movement: load and speed invariance of relative joint torques. Journal of Neurophysiology, 1996.

[10] Viviani P. and Flash T. Minimum-jerk, two-thirds power law, and isochrony: converging approaches to movement planning. J Exp Psychol Hum Percept, Feb 1995.

[11] B. Corteville, E. Aertbelien, H. Bruyninckx, J. De Schutter, and H. Van Brussel. Human-inspired robot assistant for fast point-to-point movements. In EuroHaptics Conference, 2007 and Symposium on Haptic Interfaces for Virtual Environment and Teleoperator Systems. World Haptics 2007., volume 1, pages 446-452, Mar 2007.

[12] Ariff G., Donchin O., Nanayakkara T., and Shadmehr R. A real-time state predictor in motor control: study of saccadic eye movements during unseen reaching movements. The Journal of Neuroscience, 22, Sept 2002.

[13] Roland S. Johansson, Gran Westling, Anders Bckstrm, and J. Randall Flanagan. Eye-hand coordination in object manipulation . The Journal of Neuroscience, 21:6917-6932, Sept 2001.

[14] K. Sakita, K. Ogawara, S. Murakami, K. Kawamura, and K. Ikeuchi. Flexible cooperation between human and robot by interpreting human intention from gaze information. In Intelligent Robots and Systems, 2004. (IROS 2004). Proceedings. 2004 IEEE/RSJ International Conference on, volume 1, pages 846-851, Sept 2004

[15] Kyuwan Choi, H. Hirose, T. Iijima, and Y. Koike. Prediction of four degrees of freedom arm movement using emg signal. In Engineering in Medicine and Biology Society, 2005. IEEE-EMBS 2005. 27th Annual International Conference of the, pages 5820-5823, 2005.

[16] C. Fleischer, C. Reinicke, and G. Hommel. Predicting the intended motion with emg signals for an exoskeleton orthosis controller. In Intelligent Robots and Systems, 2005. (IROS 2005). 2005 IEEE/RSJ International Conference on, pages 2029 - 2034, Aug 2005.

[17] V. Duchaine and C. M. Gosselin. Human-inspired robot assistant for fast point-to-point movements. In Robotics and Automation, 2007 IEEE International Conference on, pages 3639-3644, Apr 2007. 